\title{
Energy transport in the Vaidya system
}

\author{
J. P. Krisch and E. N. Glass \\ Department of Physics, University of Michigan, Ann Arbor, Michigan 48109
}

(Received 15 February 2005; accepted 18 March 2005; published online 16 May 2005)

\begin{abstract}
Energy transport mechanisms can be generated by imposing relations between null tetrad Ricci components. Several kinds of mass and density transport generated by these relations are studied for the generalized Vaidya system. (C) 2005 American Institute of Physics. [DOI: 10.1063/1.1915290]
\end{abstract}

\section{INTRODUCTION}

The Vaidya ${ }^{1}$ space-time generalized the Schwarzschild vacuum solution by allowing mass parameter $m_{0}$ to be a function of retarded time. The extension created a spherically symmetric null fluid atmosphere. Glass and $\mathrm{Krisch}^{2}$ pointed out that allowing the mass function to also depend on the radial coordinate created a more complex atmosphere containing an anisotropic string fluid in addition to Vaidya radiation.

The fluid parameters for the Vaidya atmosphere $\left\{\rho, p_{r}, p_{\perp}\right\}$ all depend on time through their relationship to the time-dependent mass function $m(u, r)$; they control the motion of matter through the atmosphere. The Ricci tensor null tetrad components (i.e., Ricci spinor components), $\Phi_{11}$ and $\Phi_{22}$, for the generalized Vaidya space-time also depend on both spatial and time derivatives of the mass function. If we impose relations between these components then those relations will, in turn, impose matter transport in the Vaidya system. Conversely, if a particular mode of matter transport were assumed for the mass or one of the fluid parameters, it would contain within it a Ricci relation. Relating Ricci components is a way of imposing and classifying a range of atmospheric matter transport mechanisms. In this paper we consider the generalized Vaidya metric, ${ }^{2,3}$ and examine how functional relations between $\Phi_{11}$ and $\Phi_{22}$ lead to mass transport described by the diffusion equation, by the wave equation, and by a dissipative transport equation of Telegrapher type.

In studying fluid transport, the transport equations are often reducible to ordinary differential equations by introducing a similarity variable; a well-known example of this is the diffusion variable, $\eta \sim r / \sqrt{t}$, suggested by Boltzmann ${ }^{4}$ in 1894 . All the transport equations studied in this paper have similarity solutions. The physical similarity of matter fields has been discussed by many authors. ${ }^{5-8}$ The matter transport considered here is in the atmosphere around a compact object or an already existing black hole. By examining the similarity structure of the mass solution to the transport equations, we are able to relate the function of proportionality between the Ricci components to the spatial part of a similarity variable.

The paper is structured as follows: In the next section we briefly review the generalized Vaidya space-time. Matter transport mechanisms and their similarity structure are discussed in Sec. III. Some explicit examples are given in Sec. IV. Metric and tetrad details are provided in the Appendix.

Our sign conventions are $2 A_{\nu ;[\alpha \beta]}=A_{\mu} R_{\nu \alpha \beta}^{\mu}, R_{\mu \nu}=R_{\mu \nu \alpha}^{\alpha}$, and metric signature $(+,-,-,-)$. Greek indices range over $(0,1,2,3)=(u, r, \vartheta, \varphi) . \dot{m}$ abbreviates $\partial m / \partial u, m^{\prime}$ abbreviates $\partial m / \partial r$, with another prime for each higher derivative. Overhead carets denote unit vectors. Field equations are $G_{\mu v}=-8 \pi T_{\mu v}$.

\section{GENERALIZED VAIDYA SPACE-TIME}

The Vaidya metric generalizes vacuum Schwarzschild when $m_{0} \rightarrow m(u)$, 


$$
g_{\alpha \beta}^{\mathrm{vad}} \mathrm{d} x^{\alpha} \mathrm{d} x^{\beta}=A^{\mathrm{vad}} \mathrm{d} u^{2}+2 \mathrm{~d} u \mathrm{~d} r-r^{2}\left(\mathrm{~d} \vartheta^{2}+\sin ^{2} \vartheta \mathrm{d} \varphi^{2}\right),
$$

where $A^{\mathrm{vad}}=1-2 m(u) / r$. The only nonzero Weyl null tetrad component is $\Psi_{2}=-m(u) / r^{3}$. The Ricci tensor, with $l_{\alpha} \mathrm{d} x^{\alpha}=\mathrm{d} u$, is

$$
R_{\alpha \beta}^{\mathrm{vad}}=\frac{2 \dot{m}}{r^{2}} l_{\alpha} l_{\beta}
$$

The Vaidya metric has been generalized so that $m(u) \rightarrow m(u, r)$ and $A^{\mathrm{vad}} \rightarrow A^{\mathrm{gv}}=1$ $-2 m(u, r) / r$,

$$
g_{\alpha \beta}^{\mathrm{gv}} \mathrm{d} x^{\alpha} \mathrm{d} x^{\beta}=A^{\mathrm{gv}} \mathrm{d} u^{2}+2 \mathrm{~d} u \mathrm{~d} r-r^{2}\left(\mathrm{~d} \vartheta^{2}+\sin ^{2} \vartheta \mathrm{d} \varphi^{2}\right) .
$$

The metric is Petrov type $\mathbf{D}$ with $l_{\mu}$ and $n_{\mu}$ principal null vectors (details are in the Appendix),

$$
\begin{gathered}
l_{\mu} \mathrm{d} x^{\mu}=\mathrm{d} u, \\
n_{\mu} \mathrm{d} x^{\mu}=\left(A^{\mathrm{gv}} / 2\right) \mathrm{d} u+\mathrm{d} r, \\
m_{\mu} \mathrm{d} x^{\mu}=-(r / \sqrt{ } 2)(\mathrm{d} \vartheta+i \sin \vartheta \mathrm{d} \varphi) .
\end{gathered}
$$

Basis vectors for the generalized Vaidya metric are the unit vector set $\left(\hat{v}_{\mu}, \hat{r}_{\mu}, \hat{\vartheta}_{\mu}, \hat{\varphi}_{\mu}\right)$ and the related null set $\left(l_{\mu}, n_{\mu}, m_{\mu}, \bar{m}_{\mu}\right)$ such that

$$
g_{\mu \nu}^{\mathrm{gv}}=\hat{v}_{\mu} \hat{v}_{\nu}-\hat{r}_{\mu} \hat{r}_{\nu}-\hat{\vartheta}_{\mu} \hat{\vartheta}_{\nu}-\hat{\varphi}_{\mu} \hat{\varphi}_{\nu}=l_{\mu} n_{\nu}+n_{\mu} l_{\nu}-m_{\mu} \bar{m}_{\nu}-\bar{m}_{\mu} m_{\nu} .
$$

In terms of the basis vectors, metric $g^{\mathrm{gv}}$ has energy-momentum ${ }^{2}$

$$
-8 \pi T_{\mu \nu}^{\mathrm{gv}}=\psi l l_{\mu} l_{\nu}+\rho \hat{v}_{\mu} \hat{v}_{\nu}+p_{r} \hat{r}_{\mu} \hat{r}_{\nu}+p_{\perp}\left(\hat{\vartheta}_{\mu} \hat{\vartheta}_{\nu}+\hat{\varphi}_{\mu} \hat{\varphi}_{\nu}\right)
$$

with components

$$
\begin{gathered}
4 \pi \psi=-\dot{m} / r^{2}, \\
4 \pi \rho=-4 \pi p_{r}=m^{\prime} / r^{2}, \\
8 \pi p_{\perp}=-m^{\prime \prime} / r .
\end{gathered}
$$

The Einstein tensor is computed from $g^{\mathrm{gv}}$ and given by

$$
G_{\mu v}^{\mathrm{gv}}=-2 \Phi_{11}\left(l_{\mu} n_{v}+n_{\mu} l_{v}+m_{\mu} \bar{m}_{v}+\bar{m}_{\mu} m_{v}\right)-2 \Phi_{22} l_{\mu} l_{v}-(\mathcal{R} / 4) g_{\mu v}^{\mathrm{gv}},
$$

with components

$$
\begin{gathered}
\Phi_{11}=\left(2 m^{\prime}-r m^{\prime \prime}\right) /\left(4 r^{2}\right)=2 \pi\left(p_{\perp}-p_{r}\right), \\
\Phi_{22}=-\dot{m} / r^{2}, \\
\mathcal{R}=2\left(r m^{\prime \prime}+2 m^{\prime}\right) / r^{2}=-16 \pi\left(p_{\perp}+p_{r}\right) .
\end{gathered}
$$

An inspection of Eq. (8) shows that a relation between $\Phi_{11}$ and $\Phi_{22}$ will generate matter transport.

$S_{\mu \nu}=\frac{1}{2}\left(R_{\mu \nu}^{\mathrm{gv}}-\frac{1}{4} \mathcal{R}^{\mathrm{gv}} g_{\mu \nu}\right)$ is the trace-free Ricci tensor. Its eigenspectrum determines the Segre type of metric $g_{\mu \nu}^{\mathrm{gv}}$. The characteristic equation, $\operatorname{det}[S-\lambda I]=0$, is 


$$
\lambda^{4}-\left(\Phi_{22}\right) \lambda^{3}-2\left(\Phi_{11}^{2}\right) \lambda^{2}+\left(\Phi_{11}^{2} \Phi_{22}\right) \lambda+\Phi_{11}^{4}=0
$$

with factors

$$
\left(\lambda-\Phi_{11}\right)\left(\lambda+\Phi_{11}\right)\left(\lambda^{2}-\Phi_{22} \lambda-\Phi_{11}^{2}\right) .
$$

This set corresponds to Segre type [2,(11)]. The Ricci relations established below will not change the Segre type.

\section{GEOMETRY AND TRANSPORT OF MATTER}

\section{A. Mass diffusion from fluid relations}

The fluid components of the energy-momentum in Eq. (6) depend on both time and spatial derivatives, so matter transport will occur. Implicit in these equations is the relation

$$
4 \pi r^{2} \dot{\rho}=\partial_{r} \dot{m} .
$$

If one also assumes

$$
\dot{m}=4 \pi D_{0} r^{2} \partial_{r} \rho
$$

then both the mass and density obey diffusion equations, the mass diffusing in a space whose determinant is dual to Vaidya, ${ }^{2}$

$$
\begin{gathered}
\dot{\rho}=D_{0} \nabla^{2} \rho, \\
\dot{m}=D_{0} \nabla^{-2} m
\end{gathered}
$$

with $\nabla^{2}=r^{-2}(\partial / \partial r) r^{2}(\partial / \partial r), \nabla^{-2}=r^{2}(\partial / \partial r) r^{-2}(\partial / \partial r)$, and $D_{0}$ the diffusion constant. Equating the nonzero Ricci components will also produce motion of the mass and density.

\section{B. Mass diffusion from Ricci components}

Consider the quantities $\left(4 r \Phi_{11}\right)$ and $\left(r^{2} \Phi_{22}\right)$. Equations (8a) and (8b) (with no assumptions) allow the relation between these quantities to be written as

$$
\partial_{u}\left(4 r \Phi_{11}\right)=r^{2} \partial_{r}\left[\frac{\partial_{r}\left(r^{2} \Phi_{22}\right)}{r^{2}}\right] .
$$

If there is a general linear relation between $\Phi_{11}$ and $\Phi_{22}$ such as

$$
\Phi_{22}=h_{d}(r) \Phi_{11},
$$

then both Ricci components will evolve diffusively. We will show in the next section that $h_{d}(r)$ is related to a spatial similarity variable for the diffusion equation. For exact solutions mass is a more physical quantity and, if we impose this relation and examine mass transport, Eqs. (8a) and (8b) imply

$$
\begin{gathered}
\Phi_{11}=-(r / 4) \partial_{r}\left(m^{\prime} / r^{2}\right), \\
\Phi_{22}=-\dot{m} / r^{2}=-\left(r h_{d} / 4\right) \partial_{r}\left(m^{\prime} / r^{2}\right) .
\end{gathered}
$$

The linear relation (13) together with (14b) yields

$$
\dot{m}=\left(r^{3} h_{d} / 4\right) \partial_{r}\left(m^{\prime} / r^{2}\right)
$$

and so the mass will also move diffusively.

The homogeneous solution of Eq. (15) is 


$$
\begin{gathered}
m=r^{3} c_{1}(u)+c_{2}(u), \quad \dot{c}_{2}+r^{3} \dot{c}_{1}=0, \\
8 \pi \rho=-8 \pi p_{r}=6 c_{1}(u), \\
p_{r}=p_{\perp} .
\end{gathered}
$$

Equation $\dot{c}_{2}+r^{3} \dot{c}_{1}=0$ is satisfied only for $c_{1}$ and $c_{2}$ both constant.

\section{Mass transport by wave motion}

The basic relation between the Ricci components, Eq. (12), is

$$
\partial_{u}\left(4 r \Phi_{11}\right)=r^{2} \partial_{r}\left[\frac{\partial_{r}\left(r^{2} \Phi_{22}\right)}{r^{2}}\right] .
$$

If one assumes the Ricci components are related by

$$
\partial_{u} \Phi_{22}=h_{w}(r) \Phi_{11}
$$

then $r^{2} \Phi_{22}$ will evolve as a wave equation solution. Focusing again on the mass behavior, Eq. (8a) for $\Phi_{11}$ and Eq. (8b) for $\Phi_{22}$ provide a wave equation for the mass,

$$
\ddot{m}-\left(h_{w} / 4\right) r^{3} m=0
$$

or

$$
\ddot{m}-\left(h_{w} / 4\right) r^{5} \partial_{r}\left(r^{-2} \partial_{r} m\right)=0 .
$$

Classical wave motion provides shape preserving traveling solutions to the wave equations for functions with argument $(k r-\omega t)$. Therefore, consider a variable of the form $\eta=R(r)-T(u)$. The wave equation becomes

$$
\left[\dot{T}^{2}-\left(h_{w} / 4\right) r^{3}\left(R^{\prime}\right)^{2}\right] m_{\eta \eta}-\left[\ddot{T}+\left(h_{w} / 4\right)\left(r^{3} R^{\prime \prime}-2 r^{2} R^{\prime}\right)\right] m_{\eta}=0 .
$$

The transport equation is

$$
\begin{gathered}
\alpha m_{\eta \eta}-\beta m_{\eta}=0, \\
\dot{T}^{2}-\left(h_{w} / 4\right) r^{3}\left(R^{\prime}\right)^{2}=\alpha, \\
\ddot{T}+\left(h_{w} / 4\right)\left(r^{3} R^{\prime \prime}-2 r^{2} R^{\prime}\right)=\beta .
\end{gathered}
$$

The simplest solutions require $\alpha$ and $\beta$ to be separately zero. These are

$$
h_{w}=\frac{4 c_{1}}{r^{3}\left(R^{\prime}\right)^{2}}, \quad R=c_{2} r^{3}+c_{3}, \quad T=T_{0} u+T_{1},
$$

and, as in diffusive transport, the proportionality function between the Ricci components is related to the similarity variable. The mass is given by any function of argument $\eta, m=F(\eta)$.

\section{Dissipative transport}

It is clear that the mode of matter transport depends on the relation chosen between $\Phi_{22}$ and $\Phi_{11}$. Dissipative waves obey a Telegrapher equation, ${ }^{9}$ combining both wave and diffusive elements, 


$$
\ddot{\chi}-c_{0}^{2} \nabla^{2} \chi+c_{1} \dot{\chi}=0
$$

where $\nabla^{2} \chi=\left(1 / r^{2}\right) \partial_{r}\left(r^{2} \partial_{r} \chi\right)$. The relationship

$$
\left(\partial_{u}+\alpha_{0}\right) \Phi_{22}+h_{t}(r) \Phi_{11}=0
$$

will generate an equation of Telegrapher form for mass,

$$
\ddot{m}-\left(h_{t} / 4\right) r \nabla^{-2} m+\alpha_{0} \dot{m}=0 .
$$

This form of causal dissipative transport provides a richer tool for modeling than a pure diffusion equation, since it avoids the infinite propagation speeds associated with parabolic diffusion equations. ${ }^{10}$ Causal transport has recently been discussed by Herrera and Santos. ${ }^{11}$ As before, the function of proportionality $h_{t}$ is related to a similarity variable.

\section{E. Similarity and $\boldsymbol{h}_{\boldsymbol{d}}$}

To see the relation of the proportionality function to the similarity structure of the diffusion equation, assume a mass solution and similarity variable with forms

$$
\begin{gathered}
m-m_{0}=T(u) F(\eta), \\
\eta=R(r) / L(u) .
\end{gathered}
$$

Rewriting the mass diffusion equation (15), with $F_{\eta}=\mathrm{d} F / \mathrm{d} \eta$ results in

$$
F_{\eta \eta}+F_{\eta}\left[L\left\{\frac{R^{\prime \prime}}{\left(R^{\prime}\right)^{2}}-\frac{2}{r R^{\prime}}\right\}+\frac{4 R}{h_{d} r\left(R^{\prime}\right)^{2}} \dot{L}\right]-\left[\frac{4}{h_{d} r\left(R^{\prime}\right)^{2}} L^{2} \frac{\dot{T}}{T}\right] F=0 .
$$

This diffusion equation should have similarity form ${ }^{4}$

$$
F_{\eta \eta}+2 \alpha \eta F_{\eta}-\beta F=0
$$

We look for solutions with the same similarity form, and so the coefficients impose constraints. The coefficient of $F$ relates $\beta$ and $h_{d}(r)$,

$$
\beta=\frac{4}{h_{d} r\left(R^{\prime}\right)^{2}} L^{2} \frac{\dot{T}}{T}
$$

Removing $r$-dependence provides the relation between $R(r)$ and $h_{d}(r)$,

$$
h_{d}=\frac{4 c_{0}}{r\left(R^{\prime}\right)^{2}} \text {. }
$$

With this $h_{d}$ constraint, the $u$-dependent part of the coefficient $\beta$ implies

$$
L^{2} \frac{\dot{T}}{T}=\beta c_{0}
$$

This allows Eq. (24) to be written as

$$
F_{\eta \eta}+F_{\eta}\left[L\left\{\frac{R^{\prime \prime}}{\left(R^{\prime}\right)^{2}}-\frac{2}{r R^{\prime}}\right\}+\left(R / c_{0}\right) \dot{L}\right]-\beta F=0 .
$$

The coefficient of $F_{\eta}$ requires 


$$
2 \alpha \eta=2 \alpha(R / L)=L\left[\frac{R^{\prime \prime}}{\left(R^{\prime}\right)^{2}}-\frac{2}{r R^{\prime}}\right]+\left(R / c_{0}\right) \dot{L} .
$$

Solutions of this equation depend on the value of $\alpha$. Some example solutions will be given in Sec. IV. We note here that the choice $R=R_{0} r^{3}+R_{1}$, which solves

$$
R^{\prime \prime} / R^{\prime}-2 / r=0,
$$

provides an analog of the Boltzman similarity variable. For this choice Eq. (28) yields

$$
L^{2}=4 c_{0} \alpha u+L_{0}^{2}
$$

with similarity variable $R(r) / L(u)$

$$
\eta=\frac{R_{0} r^{3}+R_{1}}{\sqrt{4 c_{0} \alpha u+L_{0}^{2}}}
$$

and proportionality function

$$
h_{d}=\frac{4 c_{0}}{r^{5} R_{0}^{2}}
$$

If $c_{0}=D_{0} R_{0}^{2}$ then $h_{d}=4 D_{0} / r^{5}$. This choice yields a simple diffusion equation for mass.

\section{F. Similarity and $\boldsymbol{h}_{t}$}

Assuming a mass and similarity variable of the form

$$
m-m_{0}=T(u) F(\eta), \quad \eta=R(r) / L(u)
$$

we find

$$
\begin{aligned}
& {\left[\frac{\dot{L}^{2}}{L^{2}} \frac{R^{2}}{T}-\left(\frac{h_{t}}{4}\right) r\left(R^{\prime}\right)^{2}\right] F_{\eta \eta^{-}}\left[R\left(2 \dot{L} \frac{\dot{T}}{T}+\ddot{L}-2 \frac{\dot{L}^{2}}{L}+\alpha_{0} \dot{L}\right)+\left(\frac{h_{t}}{4}\right) L\left(r R^{\prime \prime}-2 R^{\prime}\right)\right] F_{\eta}} \\
& \quad+\left[L^{2}\left(\frac{\ddot{T}}{T}+\alpha_{0} \frac{\dot{T}}{T}\right)\right] F=0 .
\end{aligned}
$$

The relationship of $h_{t}$ to the scaling variable in this similarity equation depends on the form of the time scaling function. The example that we shall use in the next section has $L=L_{0}$, and for this choice the similarity equation becomes

$$
-\frac{h_{t}}{4} r\left(R^{\prime}\right)^{2} F_{\eta \eta}+L_{0} \frac{h_{t}}{4}\left(2 R^{\prime}-r R^{\prime \prime}\right) F_{\eta}+L_{0}^{2}\left(\frac{\ddot{T}}{T}+\alpha_{0} \frac{\dot{T}}{T}\right) F=0 .
$$

For this choice of $L$, we see the diffusive relation between the proportionality function and the spatial scaling function again emerges as

$$
h_{t}=\frac{4 c_{0}}{r\left(R^{\prime}\right)^{2}} \text {. }
$$

Telegrapher transport could allow many other relations to be imposed, reflecting the richer solution structure of this transport mechanism. In the next section we give some examples. 


\section{TRANSPORT EXAMPLES}

\section{A. Similarity solutions}

As an example of the mass transport solutions based on similarity, we consider a single scenario to which we apply all three transport mechanisms. The physical setting considered is a compact object of mass $m(u, \eta)$ with an atmosphere in which transport is occurring. We use solutions where the scale variable $R$ and proportionality function $h(r)$ are the same for all transports,

$$
R=\frac{R_{0}}{3} r^{3}, \quad L=L_{0}, \quad h(r)=\frac{4 c_{0}}{R_{0}^{2} r^{5}} .
$$

In simple diffusion problems, the choice $L=L_{0}$ is applied to bounded systems where $L_{0}$ can be identified with a natural physical scale. For the Vaidya black hole, the Schwarzschild radius provides a physical distance scale and we could identify $L_{0}$ with an associated distance.

\section{Diffusive transport}

For diffusive transport with the choices above, the equations describing the evolution of the mass function are

$$
F_{\eta \eta}-\beta F=0, \quad \dot{T} / T=\beta c_{0} / L_{0}^{2} .
$$

The mass function, with time parameter $\tau_{0}^{-1}=\beta D_{0} R_{0}^{2} / L_{0}^{2}$, will be given by

$$
\begin{gathered}
m=m_{0}+T_{0} e^{u / \tau_{0}} F(\eta), \\
F(\eta)=F_{0} \sin (\sqrt{|\beta|} \eta+\delta), \quad \beta<0=F_{0} \sinh (\sqrt{\beta} \eta+\delta), \quad \beta>0 .
\end{gathered}
$$

For $\beta<0$, the atmosphere is decaying in time as the Vaidya photons carry energy out of the system, while for $\beta>0$, mass is accreting. From the field equations, the density of the atmosphere is described by

$$
\rho=\rho_{0} e^{-u / \tau_{0}} \cos (\sqrt{|\beta|} \eta+\delta)=\rho_{0} e^{u / \tau_{0}} \cosh (\sqrt{\beta} \eta+\delta),
$$

where we have identified the initial atmospheric density and a time constant for the accretion or decay

$$
\rho_{0}=\frac{T_{0} F_{0} \sqrt{|\beta|}}{2 \pi L_{0}} .
$$

The similarity variable in this example is simply a distance coordinate. The decaying solution could describe a bounded atmosphere whose density decreases with distance away from the surface. There is zero radial pressure outer boundary described by $\sqrt{|\beta|} \eta_{B}+\delta=\pi / 2$. In the decaying solutions, the atmosphere will go asymptotically to zero leaving a compact object of mass $m_{0}$. Because of the outgoing Vaidya radiation, there is no vacuum match until the atmosphere is gone. For the accreting solution, there is no zero radial pressure boundary. Since the accreting mass is entering the atmosphere from the exterior, this is expected. The density profile depends strongly on the phase $\delta$. For $\delta=0$, the density increases as one looks upward from the core surface. For nonzero $\delta$, the density decreases going up from the surface, reaching a minimum value which might be identified with the boundary between the atmosphere and the source of the accreting mass. 


\section{Telegrapher transport}

The equations for Telegrapher transport with the scaling functions chosen are

$$
\begin{gathered}
F_{\eta \eta}-\beta F=0, \\
\frac{L_{0}^{2}}{c_{0}}\left(\frac{\ddot{T}}{T}+\alpha_{0} \frac{\dot{T}}{T}\right)=\beta .
\end{gathered}
$$

Telegrapher transport has a wider set of solutions for the scaling choice than pure diffusive transport. One solution, using $\beta=0$, is

$$
\begin{gathered}
T=T_{0} e^{-\alpha_{0} u}, \\
F=F_{0} \eta, \\
m=m_{0}+\rho_{1} r^{3} e^{-\alpha_{0} u}, \quad \rho_{1}=T_{0} F_{0} / L_{0} .
\end{gathered}
$$

The atmospheric density is related to the mass through the field equations by $\rho=m^{\prime} /\left(4 \pi r^{2}\right)$. The density for this case does not vary with radial distance from the black hole but does reflect the atmospheric decay, approaching zero as the atmosphere vanishes and the mass becomes $m_{0}$,

$$
\rho=\rho_{0} e^{-\alpha_{0} u}, \quad \rho_{0}=3 R_{0} \rho_{1} / 4 \pi .
$$

The $\beta \neq 0$ solutions are similar to the diffusive solutions,

$$
\begin{gathered}
T=T_{0} e^{\gamma u}, \quad \beta=\left(L_{0}^{2} / c_{1}\right)\left(\gamma^{2}+\alpha_{0} \gamma\right), \\
F=F_{0} \sin (\sqrt{|\beta|} \eta+\delta), \quad \beta<0, \\
F=F_{0} \sinh (\sqrt{\beta} \eta+\delta), \quad \beta>0, \\
m=m_{0}+T(u) F(\eta) .
\end{gathered}
$$

\section{B. A diffusively evaporating atmosphere}

(a) The boundary behavior: In this example, we consider a compact object whose atmosphere is diffusively evaporating. An exact mass solution, given in Ref. 2, is

$$
m=m_{0}+(4 \pi / 3) r^{3} \rho_{0}-(4 \pi / 3) k_{2}\left(r^{5} / 10+\mathfrak{D}_{0} u r^{3}\right) .
$$

For this example the proportionality function follows directly from the diffusion equation:

$$
h_{d}=4 D_{0} / r .
$$

The atmospheric density associated with this mass is

$$
\rho=\rho_{0}-k_{2}\left(r^{2} / 6+\mathfrak{D}_{0} u\right) .
$$

A boundary can be defined by requiring the radial pressure (and density, from the equation of state) to be zero. Solving the density equation for a boundary radius gives the boundary as a function of retarded time, 


$$
R_{b}^{2}=\frac{6}{k_{2}}\left(\rho_{0}-k_{2} \mathfrak{D}_{0} u\right) .
$$

Let the boundary move inward as the atmosphere evaporates until the atmosphere is gone and the radius is at the compact object boundary, $R_{c}$. This happens in time $u_{0}$, thus

$$
R_{c}^{2}=\frac{6}{k_{2}}\left(\rho_{0}-k_{2} \mathfrak{D}_{0} u_{0}\right) .
$$

It follows that

$$
R_{b}^{2}(u)=R_{c}^{2}+6 \mathfrak{D}_{0}\left(u_{0}-u\right) .
$$

The bounding surface of the core is parametrized as $R_{c}=2 m_{0} \alpha$. Substituting into (43) provides

$$
R_{b}^{2}(u)=4 m_{0}^{2} \alpha^{2}+6 \mathfrak{D}_{0}\left(u_{0}-u\right) .
$$

The mass function places a constraint on the parameters. From Eq. (42) at time $u_{0}$,

$$
(\alpha-1) m_{0}=\frac{128}{45} k_{2} \pi m_{0}^{5} \alpha^{5} .
$$

Using Eqs. (42) and (45), the time for the evaporation process to complete is

$$
\mathfrak{D}_{0} u_{0}=\frac{2}{3} \alpha^{2} m_{0}^{2}\left[\frac{64 \pi \rho_{0} m_{0}^{2} \alpha^{3}}{15(\alpha-1)}-1\right] \text {. }
$$

The density and mass evolve as

$$
\begin{gathered}
\rho=\frac{k_{2}}{6}\left(R_{c}^{2}-R_{b}^{2}\right)+k_{2} \mathfrak{D}_{0}\left(u_{0}-u\right), \\
m=m_{0}+\frac{4 \pi k_{2}}{45} R_{b}^{5}+\frac{4 \pi}{3} k_{2} R_{b}^{3}\left[\frac{R_{c}^{2}-R_{b}^{2}}{6}+\mathfrak{D}_{0}\left(u_{0}-u\right)\right] .
\end{gathered}
$$

(b) Extremal case: The time at which the evaporation of the atmosphere is complete has an extremal value. Extremising Eq. (46) one finds

$$
\frac{2(\alpha-1)^{2}}{\alpha^{3}(4 \alpha-5)}=\frac{64 \pi \rho_{0} m_{0}^{2}}{15} .
$$

The extremal time for the evaporation is

$$
\mathfrak{D}_{0} u_{0}=\frac{2}{3} \alpha^{2} m_{0}^{2}\left[\frac{3-2 \alpha}{4 \alpha-5}\right] \text {. }
$$

The second derivative shows this is a minimum. For positive times, we require

$$
1.25<\alpha<1.5 \text {. }
$$

(c) Time estimates: Take $m_{0}$ to be a solar size, $m_{0} \sim 1.5 \times 10^{3} \mathrm{~m}$. The diffusivity constant $\mathfrak{D}_{0}$ is related to the constancy of the jump distance $(L)$ and frequency $(f) .{ }^{4} \mathfrak{D}_{0}$ can be estimated as

$$
\mathfrak{D}_{0} \sim L^{2} f \text {. }
$$

For example, if the Debye frequency in a solid is the same as the jump frequency, we would have $f \sim 10^{13} \mathrm{~Hz}$ and the jump distance could be of the order of an angstrom, so that an estimate for the diffusivity is 


$$
\mathfrak{D}_{0} \sim 10^{-7} \mathrm{~m}^{2} / \mathrm{s} .
$$

Using these estimates, the time for the atmosphere to vanish by diffusion is roughly

$$
u_{0}=10^{7}\left(\frac{2}{3}\right) \alpha^{2} \times 2.25 \times 10^{6}\left[\frac{3-2 \alpha}{4 \alpha-5}\right] \sim 1.5 \times 10^{13}\left[\frac{3-2 \alpha}{4 \alpha-5}\right] \alpha^{2} .
$$

For $\alpha=1.4$, the time is about $10^{13} \mathrm{~s} \sim 10^{5}$ years. The diffusivity is much larger than is normally measured since not all atomic oscillations will have an associated jump. A smaller diffusivity would increase the time.

\section{DISCUSSION}

In this paper we have examined some of the atmospheric matter transport mechanisms introduced by imposing a relation between Ricci components of the generalized Vaidya space-time. We found that diffusive, wave, and general Telegrapher-type transport can all follow from such a relation. Imposing a Ricci relation is a way of unifying matter transport mechanisms; if one started with a particular transport mechanism then a Ricci relation would emerge.

Examples of the matter transport mechanisms were given. All of the exact solutions describe atmospheres that are either accreting or decaying. The first two examples had the same similarity function but the density profiles in each solution were very different. The first Telegrapher solution describes a core object with a decaying atmospheric density varying only with time, and whose tangential and radial stress are all spatially constant tensions. In the pure diffusive solution, the densities vary with both distance from the core surface and time. The decaying atmospheres could be bounded by an exterior fluid of Vaidya radiation. The accreting solutions were surrounded by the source of accreting matter and Vaidya radiation. The third example, with a different proportionality function, examined a diffusing atmosphere around a core object of radius $R_{c}$ in the range $2.5 m_{0}<R_{c}<3 m_{0}$. For an $R_{c}=2.8 m_{0}$ object with a diffusivity based on a solid Debye frequency there was a minimum evaporation time of about $10^{5}$ years. Jump frequencies based on the actual atmospheric fluid would increase this time. Apart from the actual size of the evaporation time for a specific object, the model predicts that atmospheres around smaller core objects, near the lower end of the range, will take very much longer to diffusively evaporate than the atmospheres of larger core objects. This can be understood by looking at the initial size of the atmosphere. Using Eq. (44) at $u=0$, Eq. (49), and the parametrized boundary surface $R_{c}=2 m_{0} \alpha$, the initial atmospheric radius is

$$
\left.R_{b}^{2}\right|_{u=0}=2 R_{c}^{2}\left[\frac{\alpha-1}{4 \alpha-5}\right], \quad 1.25<\alpha<1.5 .
$$

The smaller core objects have larger atmospheric envelopes, taking longer to evaporate. The smaller core has an extended atmosphere because its gravitational field is not strong enough to hold the atmosphere compactly.

Some additional insights into the meaning of the proportionality function can be seen for diffusive and wave transport by writing the transport equations in terms of variable $w=r^{3}$. The diffusive and wave transport equations become

$$
\begin{aligned}
& \dot{m}=\left(\frac{9 r^{5} h_{d}}{4}\right) m_{w w}, \\
& \ddot{m}=\left(\frac{9 r^{5} h_{w}}{4}\right) m_{w w} .
\end{aligned}
$$

In each case we can find the functional form of $h(r)$ that will produce the simplest transport and can relate that value to the diffusion constant or the wave velocity. Our example solutions include this simple case and a case where the mass and density evolution are more complex. 
The choice $h_{d}(r)=4 D_{0} / r^{5}$ yields a simple $(u, w)$ diffusion equation for mass. From the Ricci relations and the field equations we see that

$$
\dot{m} / r^{2}=2 \pi h_{d}\left(p_{r}-p_{\perp}\right)
$$

indicating that, with the Ricci relations, mass transport is driven by the pressure anisotropy. This also illustrates a drawback of the mass transport ansatz. $\Phi_{11}$ is certainly zero for isotropic pressures with a string equation of state [Eq. (8a) with $p_{\perp}=p_{r}$ ]. The assumed relation, $\Phi_{22}$ $=h_{d}(r) \Phi_{11}$, then says that there is no time variation in the mass for isotropic pressures. In general, when the two Ricci components are not related, the mass can vary with time, even in the case of isotropic pressures.

We list the assumptions that lead to matter transport. For diffusion the specific choice $h_{d}$ $=4 D_{0} / r^{5}$ is used.

Assumption

$\dot{m}=4 \pi D_{0} r^{2} \partial_{r} \rho$
$\Phi_{22}=\left(4 D_{0} / r^{5}\right) \Phi_{11}$
$\partial_{u} \Phi_{22}=h_{w}(r) \Phi_{11}$
$\left(\partial_{u}+\alpha_{0}\right) \Phi_{22}+h_{t}(r) \Phi_{11}=0$
Transport

$$
\begin{aligned}
& \dot{m}-D_{0} \nabla^{-2} m=0, \dot{\rho}-D_{0} \nabla^{2} \rho=0 \\
& \dot{m}-D_{0} \nabla^{-2} m=0 \\
& \ddot{m}-\left(h_{w} / 4\right) r^{3} \nabla^{-2} m=0 \\
& \ddot{m}-\left(h_{t} / 4\right) r \nabla^{-2} m+\alpha_{0} \dot{m}=0
\end{aligned}
$$

We have seen that imposing a Ricci relation provides a broad arena to investigate a range of atmospheric transport processes in the generalized Vaidya space-time and is a rich source of new analytic mass solutions. The mass solutions that we presented focused on the growth or depletion of an atmosphere around a central object. They can be used to describe the behavior, for example, of isolated black hole atmospheres but also offer simple models of galaxies with a massive black hole at the center. The relationships we imposed on the Ricci components were investigated in terms of mass transport although the fundamental relationships described the evolution of the Ricci components themselves. The Ricci evolution is an interesting avenue for further investigation as they are input functions for the Riemann invariants. The evolution of the invariants and their syzigies will be discussed elsewhere.

\section{APPENDIX: GENERALIZED VAIDYA PRINCIPAL NULL FRAME}

The principal null frame Eq. (3) of the Petrov type D metric $g_{\mu \nu}^{\mathrm{gv}}$ obeys

$$
\begin{gathered}
l_{\mu ; \nu}=\left(A_{\mathrm{gv}}^{\prime} / 2\right) l_{\mu} l_{\nu}-(1 / r)\left(m_{\mu} \bar{m}_{\nu}+\bar{m}_{\mu} m_{\nu}\right), \\
n_{\mu ; \nu}=-\left(A_{\mathrm{gv}}^{\prime} / 2\right) n_{\mu} l_{\nu}+\left(A^{\mathrm{gv}} / 2 r\right)\left(m_{\mu} \bar{m}_{\nu}+\bar{m}_{\mu} m_{\nu}\right), \\
m_{\mu ; \nu}=\left(A^{\mathrm{gv}} / 2 r\right) l_{\mu} m_{\nu}-(1 / r) n_{\mu} m_{\nu}+(\cot \vartheta / \sqrt{2} r)\left(m_{\mu} m_{\nu}-m_{\mu} \bar{m}_{\nu}\right) .
\end{gathered}
$$

with both principal null vectors $l_{\mu}$ and $n_{\mu}$ geodesic. by

For tetrad $\{\hat{v}, \hat{r}, \hat{\vartheta}, \hat{\varphi}\}$ and metric $g_{\mu \nu}^{\mathrm{gv}}=\hat{v}_{\mu} \hat{v}_{\nu}-\hat{r}_{\mu} \hat{r}_{\nu}-\hat{\vartheta}_{\mu} \hat{\vartheta}_{\nu}-\hat{\varphi}_{\mu} \hat{\varphi}_{\nu}$, the basis vectors are related

$$
\begin{gathered}
\hat{v}_{\mu} \mathrm{d} x^{\mu}=A_{\mathrm{gv}}^{1 / 2} \mathrm{~d} u+A_{\mathrm{gv}}^{-1 / 2} \mathrm{~d} r=A_{\mathrm{gv}}^{-1 / 2}\left[n_{\mu}+\left(A_{\mathrm{gv}} / 2\right) l_{\mu}\right] \mathrm{d} x^{\mu}, \\
\hat{r}_{\mu} \mathrm{d} x^{\mu}=A_{\mathrm{gv}}^{-1 / 2} \mathrm{~d} r=A_{\mathrm{gv}}^{-1 / 2}\left[n_{\mu}-\left(A_{\mathrm{gv}} / 2\right) l_{\mu}\right] \mathrm{d} x^{\mu}, \\
\hat{\vartheta}_{\mu} \mathrm{d} x^{\mu}=r \mathrm{~d} \vartheta=(1 / \sqrt{ } 2)\left(m_{\mu}+\bar{m}_{\mu}\right) \mathrm{d} x^{\mu},
\end{gathered}
$$




$$
\hat{\varphi}_{\mu} \mathrm{d} x^{\mu}=r \sin \vartheta \mathrm{d} \varphi=-(i / \sqrt{ } 2)\left(m_{\mu}-\bar{m}_{\mu}\right) \mathrm{d} x^{\mu} .
$$

The kinematics of the $\hat{v}$ flow, acceleration, expansion, and shear, are described by

$$
\hat{v}_{; \nu}^{\mu}=a^{\mu} \hat{v}_{\nu}+\sigma_{\nu}^{\mu}-(\Theta / 3)\left(\hat{r}^{\mu} \hat{r}_{\nu}+\hat{\vartheta}^{\mu} \hat{\vartheta}_{\nu}+\hat{\varphi}^{\mu} \hat{\varphi}_{\nu}\right)
$$

where

$$
\begin{gathered}
a^{\mu}=\left[\dot{m} / r+A_{\mathrm{gv}} \partial_{r}(m / r)\right] A_{\mathrm{gv}}^{-3 / 2} \hat{r}^{\mu}, \\
\sigma_{\nu}^{\mu}=(\Theta / 3)\left(-2 \hat{r}^{\mu} \hat{r}_{\nu}+\hat{\vartheta}^{\mu} \hat{\vartheta}_{\nu}+\hat{\varphi}^{\mu} \hat{\varphi}_{\nu}\right), \\
\Theta=(\dot{m} / r) A_{\mathrm{gv}}^{-3 / 2} .
\end{gathered}
$$

Spherical symmetry allows the function $m(u, r)$ to be identified as the mass within two surfaces of constant $u$ and $r$, and invariantly defined from the sectional curvature of those surfaces,

$$
-2 m / r^{3}=R_{\alpha \beta \mu \nu} \hat{\vartheta}^{\alpha} \hat{\varphi}^{\beta} \hat{\vartheta}^{\mu} \hat{\varphi}^{\nu}
$$

${ }^{1}$ P. C. Vaidya, Nature (London) 171, 260 (1953).

${ }^{2}$ E. N. Glass and J. P. Krisch, Class. Quantum Grav. 16, 1175 (1999).

${ }^{3}$ E. N. Glass and J. P. Krisch, Phys. Rev. D 57, R5945 (1998).

${ }^{4}$ R. Ghez, A Primer of Diffusion Problems (Wiley, New York, 1988).

${ }^{5}$ E. N. Glass and J. P. Krisch, J. Math. Phys. 40, 4056 (1999).

${ }^{6}$ B. Carter and R. N. Henriksen, J. Math. Phys. 32, 2580 (1991).

${ }^{7}$ A. A. Coley, Class. Quantum Grav. 14, 87 (1997).

${ }^{8}$ I. Yavuz and I. Yilmaz, Gen. Relativ. Gravit. 29, 1295 (1997).

${ }^{9}$ P. Rosenau, Phys. Rev. E 48, R655 (1993).

${ }^{10}$ D. D. Joseph and L. Preziosi, Rev. Mod. Phys. 61, 41 (1989).

${ }^{11}$ L. Herrera and N. O. Santos, gr-qc/0410014 (2004). 